This is the peer reviewed version of the following article:

Li J., Xu X., Luo Z., Zhang C., Zuo Y., Zhang T., Tang P., Infante-Carrió M.F., Arbiol J., Llorca J., Liu J., Cabot A.. Co-Sn Nanocrystalline Solid Solutions as Anode Materials in Lithium-I on Batteries with High Pseudocapacitive Contribution. ChemSusChem, (2019). 12. : 1451 - . 10.1002/cssc. 201802662,

which has been published in final form at https://dx.doi.org/10.1002/cssc. 201802662. This article may be used for non-commercial purposes in accordance with Wiley Terms and Conditions for Use of Self-Archived Versions. 


\title{
Co-Sn nanocrystalline solid solutions as anode materials in lithium-ion batteries with high pseudocapacitive contribution
}

\author{
Junshan Li, ${ }^{[a, b, o]}$ Xijun Xu, ${ }^{[c, d, o]}$ Zhishan Luo, ${ }^{[a]}$ Chaoqi Zhang, ${ }^{[a]}$ Yong Zuo, ${ }^{[a]}$ Ting Zhang, ${ }^{[e]}$ Pengyi \\ Tang, ${ }^{[e]}$ Maria F. Infante-Carrió, ${ }^{[e]}$ Jordi Arbiol,,${ }^{[e, f]}$ Jordi Llorca, ${ }^{[g]}$ Jun Liu, ${ }^{\left[c, d,{ }^{*}\right]}$ Andreu Cabott ${ }^{\left[a, f,{ }^{*}\right]}$
}

\begin{abstract}
Co-Sn solid-solution nanoparticles with the Sn crystal structure and tuned metal ratios were synthesized by a facile onepot solution-based procedure involving the initial reduction of a Sn precursor and the posterior incorporation of Co within the Sn lattice. Such nanoparticles were used as anode materials for Li-ion batteries. Among the different compositions tested, $\mathrm{Co}_{0.7} \mathrm{Sn}$ and $\mathrm{Co}_{0.9} \mathrm{Sn}$ electrodes provided the highest capacities, with values above 1500 mAh g-1 at a current density of $0.2 \mathrm{~A} \mathrm{~g}^{-1}$ after 220 cycles and up to $800 \mathrm{mAh} \mathrm{g}^{-1}$ at $1.0 \mathrm{~A} \mathrm{~g}^{-1}$ after 400 cycles. Up to $81 \%$ pseudocapacitance contribution at a sweep rate of $1.0 \mathrm{mV} \mathrm{s}^{-1}$ were measured for these electrodes, providing fast kinetics and long durability. The excellent performance of Co-Sn alloy nanoparticlebased electrodes was associated to both the small size of the crystal domains and their suitable composition, which buffered volume changes of $\mathrm{Sn}$ and contributed to a suitable electrode restructuration.
\end{abstract}

\section{Introduction}

Lithium-ion batteries (LIBs) have become the dominant energy storage technology in portable applications. However, their energy density, charging rate, and stability are still critical performance parameters that has plenty of room for improvement by optimizing both anode and cathode materials. ${ }^{[1-}$ ${ }^{4]}$ At the anode, current commercial LIBs use graphite, which has a relatively low maximum theoretical capacity $\left(372 \mathrm{mAh} \mathrm{g}^{-1}\right.$ to form $\left.\mathrm{LiC}_{6}\right) .{ }^{[5]}$ Alternatively, anode materials such as $\mathrm{Sn}, \mathrm{Ge}$ and Si provide platforms with potentially higher lithium storage capacities: $\mathrm{Li}_{22} \mathrm{Sn}_{5}\left(994 \mathrm{mAh} \mathrm{g}^{-1}\right), \mathrm{Li}_{22} \mathrm{Ge}_{5}\left(1600 \mathrm{mAh} \mathrm{g}^{-1}\right)$, and $\mathrm{Li}_{22} \mathrm{Si}_{5}$ (3000 mAh g${ }^{-1}$ ) respectively. ${ }^{[6-8]}$ While $\mathrm{Si}$ is the most abundant element and has the highest potential storage capacity, Sn and Sn-based compounds are particularly appealing owing to

[a] Catalonia Institute for Energy Research - IREC, Sant Adrià de Besòs, Barcelona, 08930, Spain. *Prof. A. Cabot, acabot@irec.cat

[b] Departament d'Electronica, Universitat de Barcelona, 08028 Barcelona, Spain

[c] Guangdong Provincial Key Laboratory of Advanced Energy Storage Materials, School of Materials Science and Engineering, South China University of Technology, Guangzhou 510641, P. R. China.*Prof. J. Liu, msjliu@scut.edu.cn

[d] SUNWODA-SCUT Joint Laboratory for Advanced Energy Storage Technology, South China University of Technology, Guangzhou 510641, P. R. China

[e] Catalan Institute of Nanoscience and Nanotechnology (ICN2), CSIC and BIST, Campus UAB, Bellaterra, 08193 Barcelona, Spain

[f] ICREA, Pg. Lluís Companys 23, 08010 Barcelona, Spain

[g] Institute of Energy Technologies, Department of Chemical Engineering and Barcelona Research Center in Multiscale Science and Engineering. Universitat Politècnica de Catalunya, EEBE, 08019 Barcelona, Spain

$\mathrm{O}$ J. Li and X. Xu equally contributed to this work.

Supporting information for this article is given via a link at the end of the document. their relatively high abundance, low cost and high electrical conductivity. ${ }^{[7,9]}$

In terms of stability, the huge lattice expansion and contraction of the anode material during cycling strongly reduces the battery performance due to a loss of electrical connection by electrode pulverization. In the case of $\mathrm{Sn}$, the $\mathrm{Sn} / \mathrm{Li}_{22} \mathrm{Sn}_{5}$ reaction has associated a $300 \%$ volume change, which inevitably leads to major structural rearrangements generally resulting in a loss of capacity. ${ }^{[10,11]}$ Different strategies have been proposed to tackle down this major issue. One main approach is to alloy the active phase, $\mathrm{Sn}$, with a second elements that buffers the volume changes. ${ }^{[12,13]}$ In this direction, Sn-based alloys with $\mathrm{Ni},{ }^{[14-17]} \mathrm{Co},{ }^{[18-30]} \mathrm{Fe},{ }^{[11,32]} \mathrm{Cu},{ }^{[33,34]}$ and $\mathrm{Sb}^{[35-37]}$ have demonstrated superior cycling performance than bare $\mathrm{Sn}$ anodes. Among the different Sn-based alloys tested, CoSn electrodes have shown particularly promising performances as anode materials for LIBs. ${ }^{[31]}$ Co-Sn-C composites have been even used in commercial batteries, what has motivated a notable interest in this system. ${ }^{[38-42]}$ Most previous works have focused on the intermetallic Co-Sn alloys: $\mathrm{Co}_{3} \mathrm{Sn}_{2}, \mathrm{CoSn}, \mathrm{CoSn}$. Among these intermetallics, while some controversy remains, $\mathrm{CoSn}_{2}$ has been considered as the optimum stoichiometry, due to its highest content of Sn. However, beyond intermetallic phases, a range of Co-Sn solid solutions exist that are yet to be explored.

Besides alloying the active material to improve cycling performance, the use of nanostructured electrodes can reduce the overall stress generated on the material domains during lithiation, thus diminishing mechanical disintegration and improving stability. Furthermore, nanosized materials present additional advantages, such as faster rate capabilities because of the shorter Li-ion diffusion paths and a potentially huge pseudocapacitive contribution associated with their very high surface/volume ratio. ${ }^{[43-46]}$ This pseudocapacitive contribution is particularly appealing because it can significantly improve both the rate performance of LIBs and their stability.

In the present work, we take advantage of the versatility of colloidal synthesis methods to produce Co-Sn solid solution nanoparticles (NPs) with tuned Co:Sn ratios, from 0.3 to 1.3. After removing surface ligands, we use these NPs to test the performance of Co-Sn solid solutions as anode materials for LIBs, defining an optimal composition and demonstrating this system to be characterized by a high energy storage capacity, with a high pseudocapacitive contribution and a notable stability.

\section{Results and Discussion}

Colloidal Co-Sn NPs were produced by the reduction of $\operatorname{tin}($ II) acetate and cobalt(II) acetylacetonate with borane tertbutylamine (TBAB) at $180^{\circ} \mathrm{C}$, in a solution containing oleylamine $(\mathrm{OAm})$, oleic acid (OAc) and tri-n-octylphosphine (TOP, see details in experimental section). Figures 1a-d show representative TEM micrographs and size distribution 
histograms of the quasi-spherical NPs produced. The average NP size was estimated to be $6-7 \mathrm{~nm}$ for all compositions except for the Sn-richest NPs, which had an average size of $10 \mathrm{~nm}$. $\mathrm{XRD}$ analysis showed that, independently of the Co:Sn ratio, NPs conserved the Sn crystal structure. However, XRD peaks appeared shifted to lower angles, as it corresponds to the introduction of a slightly larger atom, Co, within the Sn lattice. The formation of CoSn solid solutions was somehow surprising when taking into account that previous works reported the formation of intermetallics, i.e. $\mathrm{CoSn}_{2}, \mathrm{CoSn}$ and $\mathrm{Co}_{3} \mathrm{Sn}_{2}$, when co-reducing proper amounts of the two elements. We associate the differences in the products obtained between our synthesis protocol and previous works to the relatively low synthesis temperatures we used which prevented the crystallization of independent Co NPs and Co-Sn intermetallic phases.
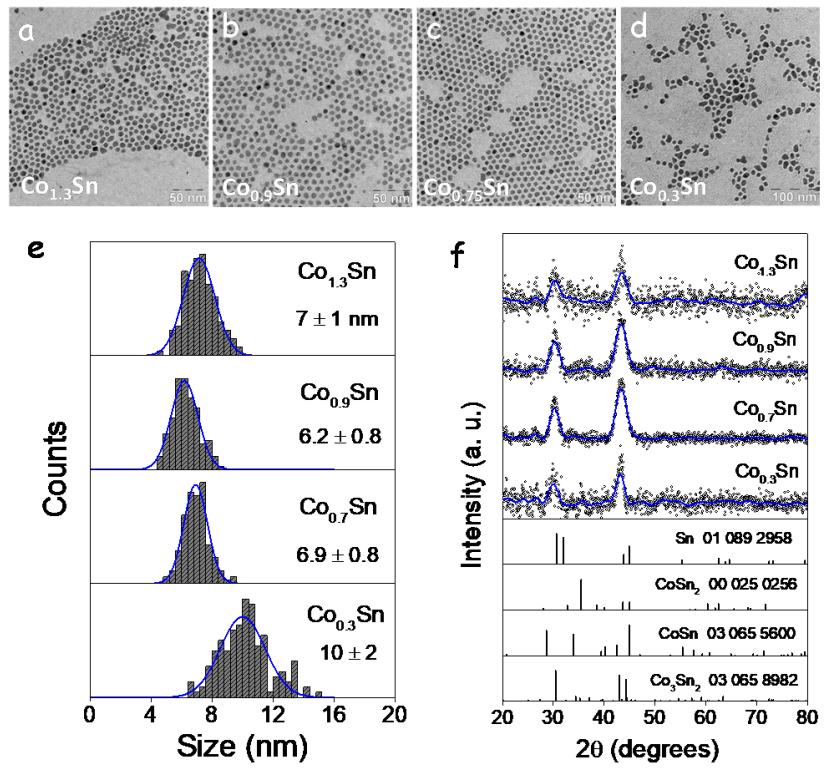

Figure 1. a-d) TEM micrographs of Co-Sn NPs with different compositions, as obtained from EDX and displayed in each image. e) Size distribution histograms of the Co-Sn NPs; f) XRD patterns of the NPs with different compositions. Sn and different Co-Sn intermetallic XRD patterns are shown as reference.

EDX analysis showed the Co:Sn ratio in Co-Sn solidsolution NPs to be: $1.3,0.9,0.7$ and 0.3 when produced from nominal Co:Sn precursor ratios of 2.0, 1.5, 1.0 and 0.5 , respectively. The final Co-poor NP stoichiometry (with respect to the nominal) and the pink color of the supernatant obtained after NP precipitation revealed that some of the cobalt precursor remained unreacted after $1 \mathrm{~h}$ at $180^{\circ} \mathrm{C}$. We also observed that the same reaction conditions but in the absence of Sn precursor did not result in the formation of Co NPs. On the contrary, the same reaction in the absence of Co resulted in the formation of Sn NPs. We believe that in the reaction conditions used, the Sn precursor was first reduced to nucleate Sn NPs. Taking advantage of the lower energy for heterogeneous growth over homogeneous nucleation, during the $1 \mathrm{~h}$ period at $180^{\circ} \mathrm{C}$ Co ions within the solution incorporated to the initial $\mathrm{Sn}$ nuclei upon reduction with TBAB. Through this synthesis mechanism, the Sn crystal structure was conserved, which is in contrast to the results obtained in previous works that make use of higher reaction, alloying or annealing temperatures to produce Co-Sn intermetallic alloys.

XPS analysis (Figure S2) showed the Co:Sn ratio of the $\mathrm{Co}_{0.9} \mathrm{Sn}$ NPs to be 0.7 , which pointed at a slightly Sn-rich surface. We hypothesize that this Sn-rich surface was related to a slight oxidation of the NPs exposed to ambient conditions. We believe air exposure resulted in a slight restructuration of the alloy due to the higher $\mathrm{Sn}$ affinity to oxygen that drove the diffusion of $\mathrm{Sn}$ to the surface. ${ }^{[4]}$

Figure 2a shows STEM micrographs and EELS chemical composition maps of the $\mathrm{Co}_{0.9} \mathrm{Sn}$ NPs. All $\mathrm{Co}_{0.9} \mathrm{Sn} \mathrm{NPs}$ contained the two elements in similar ratios. Within each NP, Co and $\mathrm{Sn}$ distributions were mostly homogeneous, but most NPs presented a Sn-rich shell, consistent with XPS analysis. HRTEM micrographs (Figure $2 \mathrm{~b}$ ) clearly displayed a core-shell structure of the NPs. From HRTEM analysis, the core crystal structure could be assigned to the $\mathrm{Co}_{2.9} \mathrm{Sn}_{2}$ orthorhombic phase (space group $=$ Pnma) with $a=7.1450 \AA, b=5.2500 \AA$ and $c=8.1730$ $\AA$, or to the $\mathrm{Co}_{3} \mathrm{Sn}_{2}$ hexagonal phase (space group $=\mathrm{P} 63 / \mathrm{mmc}$ ) with $\mathrm{a}=\mathrm{b}=4.1130 \AA$ and $\mathrm{c}=5.1850 \AA(\mathrm{SI}) \cdot{ }^{[48]}$ This result is in contradiction with XRD patterns and EDX and XPS analysis. We hypothesize that solid-solution NPs with the $\mathrm{Co}_{0.9} \mathrm{Sn}$ composition and $\mathrm{Sn}$ structure were initially formed. With the exposition to air, these NPs developed a Sn-rich surface associated to a differential reactivity of the two elements with oxygen. ${ }^{[4-51]}$ Within the electron beam during HRTEM analysis, the core, having a higher Co content due to the diffusion of $\mathrm{Sn}$ to the surface, crystallized to an intermetallic $\mathrm{Co}_{3} \mathrm{Sn}_{2}$ phase with potential additional $\mathrm{Sn}$ segregation to the surface.

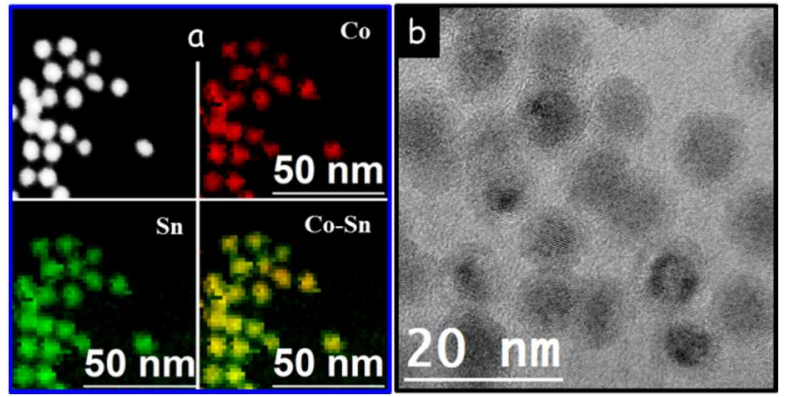

Figure 2. a) STEM and EELS compositional maps of $\mathrm{Co}_{0.9} \mathrm{Sn}$ NPs. b) HRTEM micrograph of $\mathrm{Co}_{0.9} \mathrm{Sn}$ NPs exposed to atmosphere and displaying a core-shell structure.

Co-Sn solid solution NPs were explored as anode material in LIBs. Before testing their performance, the organic ligands used to control the growth of the NPs in solution were removed by treating them with a mixture of hydrazine and acetonitrile. ${ }^{[36,37]}$ FTIR analysis confirmed the effectiveness of the ligand removal step (Figure S4). LIB anodes were prepared by casting a nonaqueous slurry containing $80 \mathrm{wt} \%$ of active material, polyvinylidene (PVDF, $10 \mathrm{wt} \%$ ) as a polymer binder, and Super $P$ as conductive additive (10 wt\%). After vacuum drying, all anodes had similar mass loading of the active materials (ca. 0.79-1.36 mg). All electrodes were tested under the same conditions, using coin type half-cells with metallic $\mathrm{Li}$ as counter electrodes (see details in experimental section). 


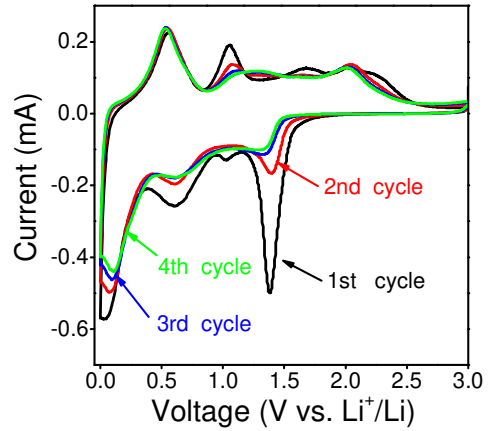

Figure 3. Initial cyclic voltammograms obtained from the $\mathrm{C}_{0.9} \mathrm{Sn}$ electrode in the voltage window $0-3.0 \mathrm{~V}$ vs. $\mathrm{Li}^{+} / \mathrm{Li}$ at $0.1 \mathrm{mV} \mathrm{s}^{-1}$.

The electrochemical performance of Co-Sn NP-based electrodes was initially assessed through cyclic voltammetry (CV) with a scan rate of $0.1 \mathrm{mV} \mathrm{s}^{-1}$ in the potential window of 0 $3.0 \mathrm{~V}$ (vs. $\mathrm{Li}^{+} / \mathrm{Li}$ ). As shown in Figure 3, all CV cycles showed a similar trend, but the initial two cycles displayed more pronounced peaks than following ones at $1.31 \mathrm{~V}$ and $2.05 \mathrm{~V}$ in the forward scan and $0.65 \mathrm{~V}$ and $1.45 \mathrm{~V}$ in the reverse one. Differences were ascribed to the formation of the solid electrolyte interphase (SEI) layer during the first cycles and the reduction of the surface $\mathrm{SnO}_{x}$ layer formed during NP manipulation and electrode preparation, in agreement with previous works. ${ }^{[52,53]}$ The overlap of the 3 rd and subsequent cycles indicated a reasonable stability of the electrode. The two obvious peaks around 0.65 and $0.05 \mathrm{~V}$ in the reverse scan were assigned to the reversible lithium insertion in the CoSn alloy to form $\mathrm{Li}_{4.4} \mathrm{Sn}^{\left[{ }^{[54]}\right.}$ During the anodic sweep, peaks at $0.52 \mathrm{~V}$ were
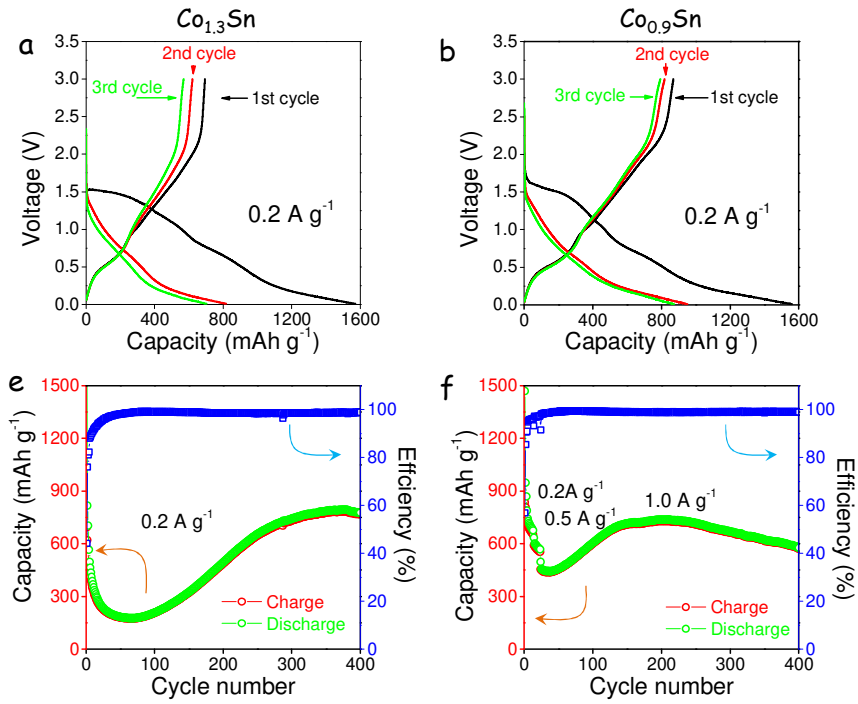

related to the extraction of $\mathrm{Li}$ ions from the electrode.
Qualitatively similar voltammograms were obtained for the four compositions tested.

Figures 4a-d display the first three charge-discharge cycles at a current density of $0.2 \mathrm{~A} \mathrm{~g}^{-1}$ of the electrodes containing Co-Sn NPs with different ratios. For all the compositions tested, small charging and discharging plateaus were observed at around $0.4 \mathrm{~V}$ and $1.6 \mathrm{~V}$, respectively. The charge voltage at ca. $0.4 \mathrm{~V}$ was in good agreement with previous results. ${ }^{[29,55]}$ Figure $4 \mathrm{e}-\mathrm{f}$ shows the charge-discharge capacity and related efficiency over 400 cycles at a current density of $1.0 \mathrm{~A} \mathrm{~g} \mathrm{~g}^{-1}$ (activated at 0.2 and $0.5 \mathrm{~A} \mathrm{~g}^{-1}$ for 10 cycles respectively). The low initial coulombic efficiency measured for all the electrodes, ca. 50\%, was associated with the SEI formation. During the first few cycles, the coulombic efficiency increased to ca. $98 \%$ and it was stabilized at this value for several hundreds of cycles. All compositions showed a similar trend, with an initial very fast decrease of the capacity, attributed to the SEI formation, a following slower loss of capacity, associated to a partial disintegration of the anode material, a capacity recover after a certain number of cycles and a moderate and sustained decrease of capacity at much larger cycle numbers. We hypothesize the reactivation to be in part associated to a rearrangement of the active material domains within the anode making a larger amount of electroactive material accessible to $\mathrm{Li}$ ions, although at the same time reducing electrical conductivity as observed from the EIS analysis below. This rearrangement of the active material could also provide larger surface areas and increase the pseudocapacitive contribution to the total energy storage capacity. ${ }^{[56]}$ On the other hand, a restructuration of the active
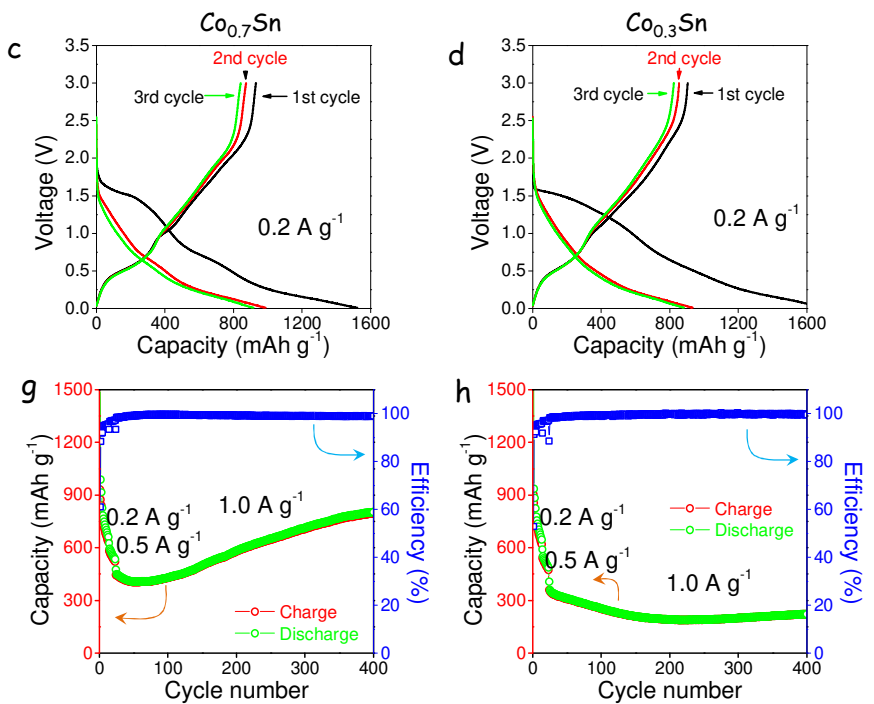

material at the atomic scale, and particularly its amorphisation, could facilitate lithium insertion.

Figure 4. a-d) Initial charge-discharge curves at $0.2 \mathrm{~A} \mathrm{~g}^{-1}$ for the different electrode compositions as displayed on the top of each graph. e-h) Charge-discharge capacity and related efficiency over 400 cycles at a current density of $1.0 \mathrm{~A} \mathrm{~g}^{-1}$ : activated at 0.2 and $0.5 \mathrm{~A} \mathrm{~g} \mathrm{~g}^{-1}$ for 10 cycles each. For the Co1.3Sn electrode only data at $0.2 \mathrm{~A} \mathrm{~g}^{-1}$ is shown

$\mathrm{Co}_{0.9} \mathrm{Sn}$ and $\mathrm{Co}_{0.7} \mathrm{Sn}$ electrodes showed the highest Li storage capacities among the compositions tested. For the $\mathrm{Co}_{0.9} \mathrm{Sn}$ electrode at $0.2 \mathrm{~A} \mathrm{~g}^{-1}$, as shown in Figure S5, during the first cycle, the coulombic efficiency was just $55.7 \%$, with a high discharge (869 mAh g $\left.{ }^{-1}\right)$ and charge capacity (1560 mAh g$\left.{ }^{-1}\right)$. A strong capacity loss was observed during the first cycles, down to charge and discharge capacities of $629 \mathrm{mAh} \mathrm{g}^{-1}$ and 647 $\mathrm{mAh} \mathrm{g}^{-1}$ with $97.2 \%$ coulombic efficiency at the $24^{\text {th }}$ cycle. With continuous cycling, the coulombic efficiency remained stable and the capacity gradually increased up to charge and discharge 
capacities of $1534 \mathrm{mAh} \mathrm{g}^{-1}$ and $1555 \mathrm{mAh} \mathrm{g}^{-1}$ at the $220^{\text {th }}$ cycle. The extraordinarily high capacities obtained, above the theoretical maximum not only for Co-Sn alloys, but also for pure $\mathrm{Sn}$, may be attributed to the ultra-small particle size of the active anode material, which provided additional active sites for Li-ion storage and a larger density of diffusion channels for $\mathrm{Li}$ ions to access all the active material. ${ }^{[56-60]}$

To evaluate the rate capability of the Co-Sn electrodes, galvanostatic cycling was performed at current rates between 0.05 to $4 \mathrm{~A} \mathrm{~g}^{-1}$ (Figure 5a). Figure $5 \mathrm{~b}$ presents the corresponding charge-discharge profiles from 0.05 to $4.0 \mathrm{~A} \mathrm{~g}^{-1}$. For $\mathrm{Co}_{0.9} \mathrm{Sn}$, the electrode delivered a discharge capacity of $804,702,598,532$, 448, 365, $267 \mathrm{mAh} \mathrm{g}^{-1}$ at 0.05, 0.1, 0.2, 0.5, 1.0, 2.0, 4.0 $\mathrm{A} \mathrm{g}^{-1}$, respectively.

Figure S6 compares the EIS data obtained from electrodes with different Co-Sn compositions and from the $\mathrm{Co}_{0.9} \mathrm{Sn}$ electrode in the first and the 400th cycle, all at $1 \mathrm{~A} \mathrm{~g}^{-1}$. From the Nyquist plots, not a straightforward dependence of the anodeelectrolyte charge transfer resistance on the Co-Sn alloy composition was observed. However, the lowest resistances were obtained for the optimum compositions $\mathrm{Co}_{0.7} \mathrm{Sn}$ and
The kinetics of the Co-Sn electrodes was further investigated using $\mathrm{CV}$ at different scan rates, from 0.1 to 1 $\mathrm{mV} \mathrm{s}^{-1}$. Figure $5 \mathrm{c}$ presents the $\mathrm{CV}$ curves obtained from the $\mathrm{Co}_{0.9} \mathrm{Sn}$ electrode at the scan rates of $0.1,0.2,0.4,0.7,1.0$ $\mathrm{mV} \mathrm{s}^{-1}$ in the potential range $0-3.0 \mathrm{~V} \mathrm{vs} \mathrm{Li}^{+} / \mathrm{Li}$. Three anodic peak were observed at $0.52,1.31,2.05 \mathrm{~V}$, all of them increasing with the scan rate.

Two main charge-storage mechanisms determine the electrode storage capacity: i) a diffusion-controlled contribution associated to the $\mathrm{Li}_{22} \mathrm{Sn}_{5}$ alloy formation; and ii) a surfacerelated capacitive contribution known as the pseudocapacitive contribution. ${ }^{[61]}$ The pseudocapacitive contribution is particularly attractive because it is a much faster and stable process, whereas the diffusion-controlled alloying is slower and generally provides relatively poor cycle life.

Generally, the relationship between the measured current $(I)$ and the scan rate $(v)$ can be expressed as:

$$
i=a v^{b}
$$

According to previous reports, a diffusion controlled process is characterized by a scan rate dependence with $b=0.5$, whereas an ideal capacitive behavior translates into $b=1 .{ }^{[62-64]}$
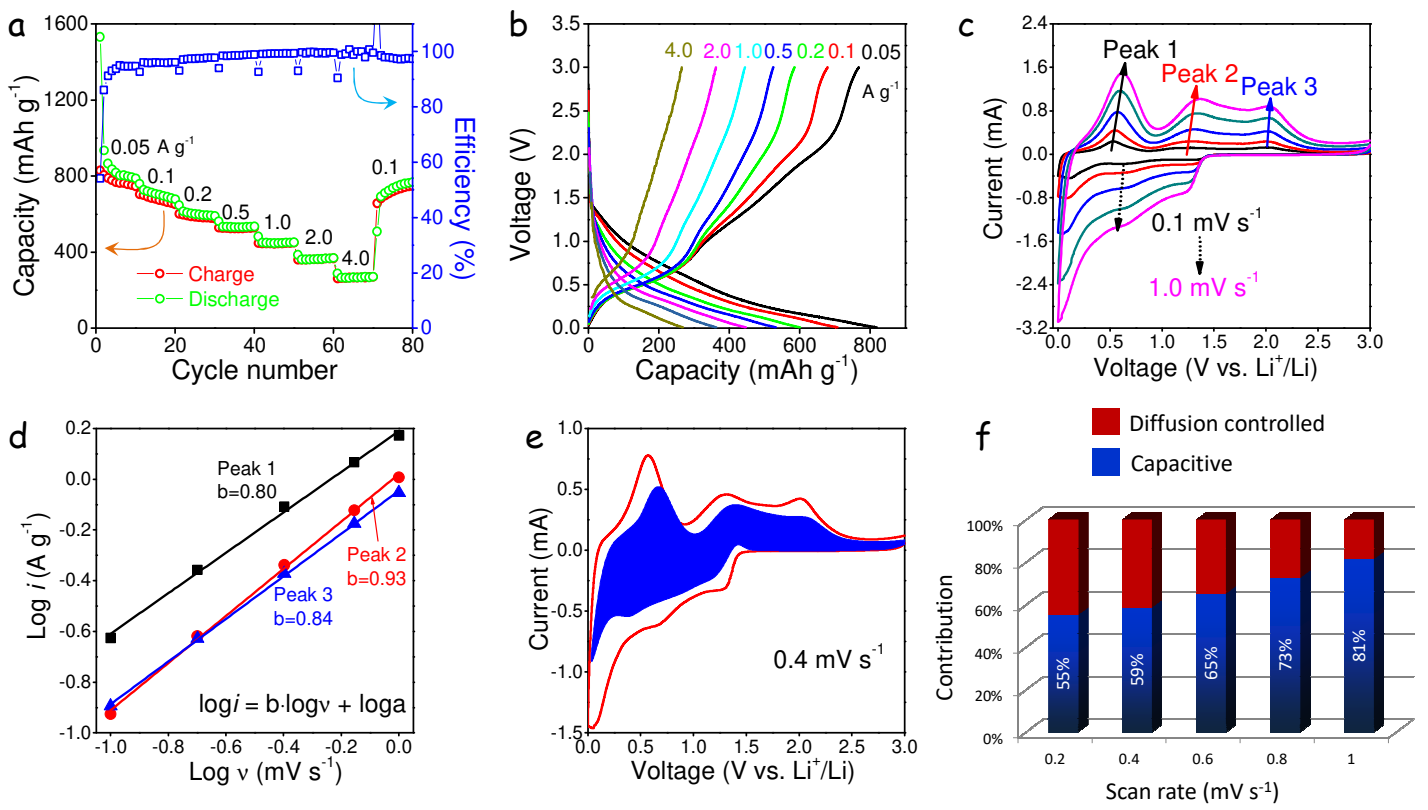

$\mathrm{Co}_{0.9} \mathrm{Sn}$. With cycling, the electrode resistance increased and two semicircles evolved, one corresponding to the SEI layer impedance and the other to the charge-transfer impedance on the electrode-electrolyte interphase. In the low frequency region, slopes well above 1 for all compositions and both for the fresh

From the linear fit of the logarithmic plot of the current vs. scan rate (Figure $5 \mathrm{~d}$ ), $b$ values of $0.80,0.93$ and 0.84 were calculated at $0.52,1.31,2.05 \mathrm{~V}$, respectively. These values indicated a fast kinetics resulting from a pseudocapacitive effect. and cycled samples indicated a significant capacitive behavior.

Figure 5. Li-ion storage performance of the Co..9Sn electrode: a) Charge-discharge curves at rates: $0.05,0.1,0.2,0.5,1.0,2.0,4.0,0.1 \mathrm{Ag}^{-1}$. b) Rate performance at $0.05,0.1,0.2,0.5,1.0,2.0,4.0 \mathrm{~A} \mathrm{~g}^{-1}$. c) $\mathrm{CV}$ curve at the scan rates of $0.1,0.2,0.4,0.7,1.0 \mathrm{mV} \mathrm{s}^{-1}$. d) Logarithmic dependence between peak current density and scan rate at the anodic peaks $0.52,1.31,2.05 \mathrm{~V}$. e) Capacitive contribution to the total current contribution at $0.4 \mathrm{mV} \mathrm{s}^{-1}$. f) Normalize capacitive and diffusion-controlled contribution at the scan rates of $0.1,0.2,0.4,0.7,1.0 \mathrm{mV} \mathrm{s}^{-1}$.

At each potential, the current density contribution at a given scan rate could be divided into two parts, a diffusioncontrolled $\left(\mathrm{k}_{1} \mathrm{v}^{1 / 2}\right)$ and a capacitor-like fraction $\left(\mathrm{k}_{2} \mathrm{v}\right)$ :

$$
i(V)=k_{1} v^{1 / 2}+k_{2} v
$$

To distinguish the fraction of the current arising from $\mathrm{Li}^{+}$ insertion and that from a capacitive process at each specific potential, $\mathrm{k}_{1}$ and $\mathrm{k}_{2}$ were determined by plotting $i(V) / v^{1 / 2} \mathrm{vs}$.

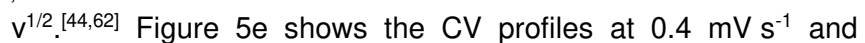
compares the capacitive current (blue shaded region) with that for the total measured current (red curve) for the $\mathrm{Co}_{0.9} \mathrm{Sn}$ electrode. The relative pseudocapacitive contributions at sweep rates of $0.1,0.2,0.4,0.7$ and $1.0 \mathrm{mV} \mathrm{s}^{-1}$ were $55 \%, 59 \%, 65 \%$, $73 \%$ and $81 \%$, respectively (Figures $4 \mathrm{f}$ and $\mathrm{S} 7$ ). For comparison, the pseudocapacitive study of $\mathrm{Co}_{0.3} \mathrm{Sn}$ electrode is presented in 
Figure S8, the calculated contributions at sweep rates of 0.1-1.0 $\mathrm{mV} \mathrm{s}^{-1}$ were lower than that of $\mathrm{Co}_{0.9} \mathrm{Sn}$. These results clearly suggest that the pseudocapacitive charge-storage amount does occupy a high portion of the whole energy storage capacity, which is associated to the small size of the Co-Sn NPs used and their Sn-rich and oxidized surface.
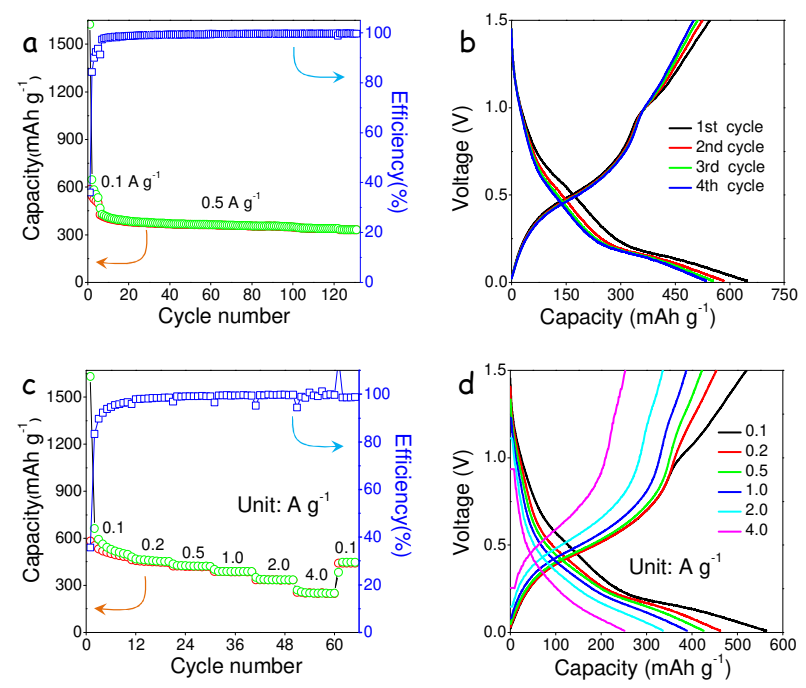

Figure 6. Li-ion storage performance of the Coo.9Sn electrode in the range 0 $1.5 \mathrm{~V}$ vs. $\mathrm{Li}^{\mathrm{Li}} \mathrm{Li}^{+}$: a) Charge-discharge curves at rates: $0.1,0.2,0.5,1.0,2.0,4.0$, $0.1 \mathrm{~A} \mathrm{~g}^{-1}$. b) Rate performance at $0.1,0.2,0.5,1.0,2.0,4.0 \mathrm{~A} \mathrm{~g}^{-1}$. c) Chargedischarge capacity and related efficiency over 130 cycles at a current density of $0.5 \mathrm{~A} \mathrm{~g}^{-1}$ : activated at $0.1 \mathrm{~A} \mathrm{~g}^{-1}$ for 10 cycles. d) Initial charge-discharge curves at $0.1 \mathrm{~A} \mathrm{~g}^{-1}$.

To estimate the practical application of Co-Sn NP-based anodes, they were tested in the range of $0.01-1.5 \mathrm{~V} \mathrm{vs}$. $\mathrm{Li} / \mathrm{Li}^{+}$. As shown in Figures 6a-b and S9, over continuous chargedischarge cycles, $\mathrm{Co}_{0.7} \mathrm{Sn}$ and $\mathrm{Co}_{0.9} \mathrm{Sn}$ electrodes show the highest capacity and stability, stabilizing at $360 \mathrm{mAh} \mathrm{g}^{-1}$ at $0.5 \mathrm{~A}$ $\mathrm{g}^{-1}$. This value is comparable to theoretical capacities of graphene-based electrodes. The rate capability of the $\mathrm{Co}_{0.9} \mathrm{Sn}$ electrode is also shown in Figure 6c-d. Specifically, the electrode delivered discharge capacities of $520,453,421,388$, $336,253 \mathrm{mAh} \mathrm{g}^{-1}$ at $0.1,0.2,0.5,1.0,2.0,4.0 \mathrm{~A} \mathrm{~g}^{-1}$, respectively. Additionally, this electrode delivered a stable charge-discharge capacity at $0.1 \mathrm{~A} \mathrm{~g} \mathrm{~g}^{-1}$ after continuous 60 cycles at variable charging rate.

\section{Conclusions}

In conclusion, Co-Sn solid-solution NPs with average size in the 6-10 $\mathrm{nm}$ range were synthesized via a simple one pot colloidalbased approach. The $\mathrm{Co}_{x} \mathrm{Sn}$ NP composition was adjusted, 1.3 $\leq x \leq 0.3$, by tuning the ratio of the initial precursors. The low synthesis temperature favoured the nucleation of Sn NPs and the subsequent inclusion of Co to the Sn lattice, forming a solid solution with the $\mathrm{Sn}$ crystal phase, instead of an intermetallic compound. The same strategy could be used to produce a much more extended range of Co-Sn compositions. Co-Sn NPs presented a Sn-rich surface after exposure to air. These Co-Sn solid solutions were tested as anode materials in LIBs on a halfcell battery system. Among the different compositions tested, $\mathrm{Co}_{0.9} \mathrm{Sn}$ and $\mathrm{Co}_{0.7} \mathrm{Sn}$ NPs provided the best performance, with a charge-discharge capacity above $1500 \mathrm{mAh} \mathrm{g}^{-1}$ at a current density of $0.2 \mathrm{~A} \mathrm{~g}^{-1}$ after 220 cycles and up to $800 \mathrm{mAh} \mathrm{g}^{-1}$ at 1.0 $\mathrm{A} \mathrm{g}^{-1}$ after 400 cycles in the range 0-3.0 $\mathrm{V}$. When testing in the range $0-1.5 \mathrm{~V}$, these two electrode delivered an average of 360 $\mathrm{mAh} \mathrm{g^{-1 }}$ at $0.5 \mathrm{~A} \mathrm{~g}^{-1}$. These values were larger than that of graphite currently used in commercial devices and larger than the theoretical maximum for Co-Sn alloys and even for pure Sn. Through the kinetic analysis of $\mathrm{Co}_{0.9} \mathrm{Sn}$ NPs by the CV measurement, we found these charge-discharge capacities to include a very large pseudocapacitive contribution, up to $81 \%$ at a sweep rate of $1 \mathrm{mV} \mathrm{s}^{-1}$, which we related to the small size of the particles.

\section{Experimental Section}

Colloidal synthesis of Sn and Co-Sn NPs: Syntheses were carried out using standard air-free techniques. All the reagents and solvent were analytical grade and used without further purification. In a typical synthesis of Co-Sn NPs with nominal composition $\mathrm{Co}_{\mathrm{S}} \mathrm{Sn}=3: 2,0.6 \mathrm{mmol}$ cobalt(II) acetylacetonate (Co(acac)2, 99\%, Sigma-Aldrich) and $0.4 \mathrm{mmol}$ tin(II) acetate $\left(\mathrm{Sn}(\mathrm{OAc})_{2}, 95 \%\right.$, Fluka) were added into a $50 \mathrm{~mL}$ threeneck round-bottomed flask. Subsequently, $20 \mathrm{~mL}$ of oleylamine (OAm, $80-90 \%, \mathrm{TCl}$ ) and $1.0 \mathrm{~mL}$ of oleic acid (OAc, Sigma-Aldrich) were loaded along with a magnetic bar in a three-neck flask connected with a thermometer, condenser and septum. The flask was heated to $80^{\circ} \mathrm{C}$ and degassed under vacuum for 2 hours and then backfilled with Ar. Then, 5 $\mathrm{mL}$ of tri-n-octylphosphine (TOP, 97\%, Strem) were injected, and afterward the solution was heated up to $180^{\circ} \mathrm{C}$ at $5{ }^{\circ} \mathrm{C} \mathrm{min}{ }^{-1}$. Right after reaching $180^{\circ} \mathrm{C}, 5 \mathrm{~mL}$ of a degassed $\mathrm{OAm}$ solution containing $5 \mathrm{mmol}$ of borane tert-butylamine (TBAB, 97\%, Sigma-Aldrich) was injected. Upon injection of this reducing complex, the solution became black, but the reaction mixture was maintained at $180^{\circ} \mathrm{C}$ for 1 additional hour to allow the NPs to grow. After $1 \mathrm{~h}$ reaction, the heating mantle was removed and the solution was cooled down to room temperature in approximately 3 min using a water bath. NPs were collected by centrifugation after adding an excess of acetone. The precipitate was dispersed in chloroform and centrifuged a second time with an excess of acetone. This washing process was repeated for three times. Finally, NPs were stored in chloroform.

Ligand removal: $25 \mathrm{~mL}$ of acetonitrile containing $0.8 \mathrm{~mL}$ hydrazine hydrated were added into a vial containing about $100 \mathrm{mg}$ of precipitated NPs. The solution was strongly stirred for 4 hours and centrifuged. The NPs were further washed with acetonitrile 3 more times, followed by vacuum-drying at room temperature. The product was kept in an Ar-filled glove box.

Characterization: X-ray diffraction (XRD) patterns were recorded at room temperature on a Bruker AXS D8 Advance X-ray diffractometer with $\mathrm{Cu} \mathrm{K}$ radiation $(\lambda=1.5106 \AA)$ operating at $40 \mathrm{kV}$ and $40 \mathrm{~mA}$. Transmission electron microscopy (TEM) analyses were performed on a ZEISS LIBRA 120, operating at $120 \mathrm{kV}$. High-resolution TEM (HRTEM) and scanning TEM (STEM) studies were carried out using a field emission gun FEI Tecnai F20 microscope at $200 \mathrm{kV}$ with a point-to-point resolution of $0.19 \mathrm{~nm}$. High angle annular dark-field (HAADF) STEM was combined with electron energy loss spectroscopy (EELS) in the Tecnai microscope by using a GATAN QUANTUM filter. Composition analysis was carried out using a ZEISS Auriga Scanning electron microscopy (SEM) equipped with an energy dispersive X-ray spectroscopy (EDS) detector operated at $20 \mathrm{kV}$. X-ray photoelectron spectroscopy (XPS) measurements were carried out in normal emission using an $\mathrm{Al}$ anode 
XR50 source operating at $150 \mathrm{~mW}$ and a Phoibos $150 \mathrm{MCD}-9$ detector. Fourier transform infrared spectrometer (FTIR) data was recorded on an Alpha Bruker spectrometer.

Electrochemical measurements: To evaluate the intrinsic electrochemical performance of Co-Sn NPs, the working electrode was prepared by mixing dried NPs, Super $\mathrm{P}$ and polyvinylidene fluoride (PVDF) with a weight ratio of 80:10:10 in an appropriate amount of $\mathrm{N}$ methy1-2-pyrrolidone (NMP) to obtain a slurry. Then, the mixture was coated onto a Cu foil. Then, it was dried in a vacuum oven at $80^{\circ} \mathrm{C}$ for 24 h. Subsequently, the foil was cut into disks with a diameter of $12 \mathrm{~mm}$. The typical mass loading of active materials was estimated to be $0.7-1.2$ $\mathrm{mg} \mathrm{cm}-2$. To test the performance of electrodes based on Co-Sn NPs, half cells were assembled in the glove box $\left(\mathrm{H}_{2} \mathrm{O}\right.$ and $\left.\mathrm{O}_{2}<0.1 \mathrm{ppm}\right)$ using

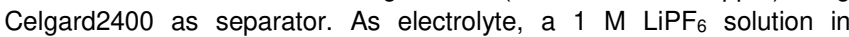
ethylene carbonate (EC)/diethyl carbonate (DEC) (1:1 in volume) with 5 wt\% fluoroethylene carbonate (FEC) as additive was used. Galvanostatic charge-discharge were measured by a battery test system (CT2001A, LAND) with cutoff potentials from $0.01 \mathrm{~V}$ to 1.5 and $3.0 \mathrm{~V}$. Cyclic voltammetry (CV) curves were performed by an electrochemical workstation (Gamry Interface 1000) in the voltage range from 0-3.0 V and the scan rate from $0.1 \mathrm{mV} \mathrm{s}^{-1}$ to $1 \mathrm{mV} \mathrm{s}^{-1}$.

\section{Author contributions}

The manuscript was prepared through the contribution of all authors. A. Cabot and J. Liu conceived and guided the project, and supervised the work. J. Li designed the experiments, produced the nanoparticles, conducted XRD, TEM, SEM-EDS and FT-IR characterization, and wrote the first draft of the manuscript. Z. Luo, C. Zhang and Y. Zuo significantly contributed to the results discussion. $X$. $X u$ performed the electrochemical measurements. T. Zhang, P. Tang, M. F. Infante-Carrió and J. Arbiol performed structural and compositional NCs characterization by means of HRTEM and EELS, and discussed the results. J. Llorca measured and discussed XPS data. The manuscript was corrected and improved by all authors.

\section{Acknowledgements}

This work was supported by the European Regional Development Funds and by the Spanish Ministerio de Economía y Competitividad through the project SEHTOP (ENE201677798-C4-3-R). J. Li thanks the China Scholarship Council for scholarship support. This project was supported by the National Natural Science Foundation of China (no. 51771076), the "1000 plan" from Chinese Government, and the Project of Public Interest Research and Capacity Building of Guangdong Province (no. 2017A010104004). TZ, PYT, MFIC and JA acknowledge funding from Generalitat de Catalunya 2017 SGR 327 and the Spanish MINECO coordinated projects between IREC and ICN2 VALPEC and subprojects RESOL and ANAPHASE (ENE201785087-C3). ICN2 acknowledges support from the Severo Ochoa Programme (MINECO, Grant no. SEV-2013-0295) and is funded by the CERCA Programme / Generalitat de Catalunya. JL is a Serra Húnter Fellow and is grateful to ICREA Academia program and to MINECO/FEDER grant ENE2015-63969-R. TZ has received funding from the CSC-UAB PhD scholarship program. Part of the present work has been performed in the framework of Universitat Autònoma de Barcelona Materials Science PhD program.

Keywords: Colloidal; Bimetallic Nanoparticles; tin cobalt; Anode; Lithium-ion Batteries

[1] C. M. Park, J. H. Kim, H. Kim, H. J. Sohn, Chem. Soc. Rev. 2010 39, 3115-3141.

[2] M. N. Obrovac, V. L. Chevrier, Chem. Rev. 2014, 114, 1144411502 .

[3] R. Marom, S. F. Amalraj, N. Leifer, D. Jacob, D. Aurbach, J. Mater. Chem. 2011, 21, 9938-9954.

[4] P. G. Bruce, B. Scrosati, J. M. Tarascon, Angew. Chemie - Int. Ed. 2008, 47, 2930-2946.

[5] L. X. Ding, G. R. Li, Z. L. Wang, Z. Q. Liu, H. Liu, Y. X. Tong, Chem - A Eur. J. 2012, 18, 8386-8391.

[6] H. Tian, F. Xin, X. Wang, W. He, W. Han, J. Mater. 2015, 1, 153169

[7] B. Wang, B. Luo, X. Li, L. Zhi, Mater. Today 2012, 15, 544-552.

[8] C. K. Chan, R. N. Patel, M. J. O'Connell, B. A. Korgel, Y. Cui, ACS Nano 2010, 4, 1443-1450.

[9] H. Ying, W. Q. Han, Adv. Sci. 2017, 4, 1700298

[10] N. Nitta, F. Wu, J. T. Lee, G. Yushin, Mater. Today 2015, 18, 252264

[11] J. B. Goodenough, K. S. Park, J. Am. Chem. Soc. 2013, 135, 1167-

[12] M. R. Palacín, Chem. Soc. Rev. 2009, 38, 2565-2575.

[13] M. F. Oszajca, M. I. Bodnarchuk, M. V. Kovalenko, Chem. Mater. 2014, 26, 5422-5432.

[14] D. wei ZHANG, C. ge YANG, J. DAI, J. wu WEN, L. WANG, C. hua CHEN, Trans. Nonferrous Met. Soc. China (English Ed. 2009, 19, 1489-1493.

[15] J. Liu, Y. Wen, P. A. Van Aken, J. Maier, Y. Yu, Nano Lett. 2014, 14, 6387-6392.

[16] X. Fan, P. Dou, A. Jiang, D. Ma, X. Xu, ACS Appl. Mater. Interfaces 2014, 6, 22282-22288.

[17] M. Uysal, T. Cetinkaya, A. Alp, H. Akbulut, J. Alloys Compd. 2015, $645,235-242$.

[18] N. Tamura, Y. Kato, A. Mikami, M. Kamino, S. Matsuta, S. Fujitani, J. Electrochem. Soc. 2006, 153, A1626.

[19] Z. Du, S. Zhang, J. Phys. Chem. C 2011, 115, 23603-23609.

[20] C. M. lonica-Bousquet, P. E. Lippens, L. Aldon, J. Olivier-Fourcade J. C. Jumas, Chem. Mater. 2006, 18, 6442-6447.

[21] G. O. Park, J. Yoon, J. K. Shon, Y. S. Choi, J. G. Won, S. Bin Park K. H. Kim, H. Kim, W. S. Yoon, J. M. Kim, Adv. Funct. Mater. 2016, 26, 2800-2808.

[22] B. O. Jang, S. H. Park, W. J. Lee, J. Alloys Compd. 2013, 574, 325330

[23] J. R. González, F. Nacimiento, R. Alcántara, G. F. Ortiz, J. L. Tirado CrystEngComm 2013, 15, 9196-9202.

[24] R. Gnanamuthu, Y. N. Jo, C. W. Lee, J. Alloys Compd. 2013, 564 95-99.

[25] G. Ferrara, C. Arbizzani, L. Damen, M. Guidotti, M. Lazzari, F. G. Vergottini, R. Inguanta, S. Piazza, C. Sunseri, M. Mastragostino, J. Power Sources 2012, 211, 103-107.

[26] J. He, H. Zhao, J. Wang, J. Wang, J. Chen, J. Alloys Compd. 2010 $508,629-635$

[27] J. Shin, W.-H. Ryu, K.-S. Park, I.-D. Kim, ACS Nano 2013, 7, 73307341.

J. Zhang, Y. Xia, J. Electrochem. Soc. 2006, 153, A1466.

[29] S. Wang, M. He, M. Walter, F. Krumeich, K. V. Kravchyk, M. V. Kovalenko, Nanoscale 2018, 10, 6827-6831.

[30] M. Walter, S. Doswald, F. Krumeich, M. He, R. Widmer, N. P. Stadie, M. V. Kovalenko, Nanoscale 2018, 10, 3777-3783.

[31] X. L. Wang, W. Q. Han, J. Chen, J. Graetz, ACS Appl. Mater. Interfaces 2010, 2, 1548-1551.

[32] L. O. Vogt, C. Villevieille, J. Mater. Chem. A 2017, 5, 3865-3874.

[33] W. X. Lei, Y. Pan, Y. C. Zhou, W. Zhou, M. L. Peng, Z. S. Ma, RSC Adv. 2014, 4, 3233-3237.

Y. M. Lin, P. R. Abel, A. Gupta, J. B. Goodenough, A. Heller, C. B. Mullins, ACS Appl. Mater. Interfaces 2013, 5, 8273-8277.

[35] Z. Liu, T. Song, U. Paik, J. Mater. Chem. A 2018, 6, 8159-8193. [36] $\quad$ M. Walter, S.

[37] M. He, M. Walter, K. V. Kravchyk, R. Erni, R. Widmer, M. V. Kovalenko, Nanoscale 2015, 7, 455-459. 
[38] J. Zhu, D. Wang, T. Liu, C. Guo, Electrochim. Acta 2014, 125, 347353.

[39] X. Liu, J. Xie, H. Zhao, P. L $\square$ K. Wang, Z. Feng, K. wierczek, Solid State lonics 2015, 269, 86-92.

[40] F. Nacimiento, P. Lavela, J. L. Tirado, J. M. Jiménez-Mateos, J. Solid State Electrochem. 2012, 16, 953-962.

[41] S. II Lee, S. Yoon, C. M. Park, J. M. Lee, H. Kim, D. Im, S. G. Doo,

H. J. Sohn, Electrochim. Acta 2008, 54, 364-369. P. Chen, L. Guo, Y. Wang, J. Power Sources 2013, 222, 526-532.

[42] P. H.-S. Kim, J. B. Cook, S. H. Tolbert, B. Dunn, J. Electrochem. Soc 2015, 162, A5083-A5090.

[44] V. Augustyn, P. Simon, B. Dunn, Energy Environ. Sci. 2014, 7, 1597-1614.

[45] T. Brezesinski, J. Wang, S. H. Tolbert, B. Dunn, Nat. Mater. 2010, 9, 146-151.

[46] L. Yang, X. Li, S. He, G. Du, X. Yu, J. Liu, Q. Gao, R. Hu, M. Zhu, J. Mater. Chem. A 2016, 4, 10842-10849.

[47] J. Li, Z. Luo, F. He, Y. Zuo, C. Zhang, J. Liu, X. Yu, R. Du, T. Zhang, M. F. Infante-Carrió, et al., J. Mater. Chem. A 2018, DOI 10.1039/C8TA08242A.

[48] H. Fjellvåg, A. Kjekshus, R. Stomberg, R. Zingales, I. Vikholm, F. Urso, J. Weidlein, R. A. Zingaro, Acta Chem. Scand. 1986, 40a, 2330.

[49] M. He, L. Protesescu, R. Caputo, F. Krumeich, M. V. Kovalenko, Chem. Mater. 2015, 27, 635-647.

[50] J. Li, Z. Luo, Y. Zuo, J. Liu, T. Zhang, P. Tang, J. Arbiol, J. Llorca, A Cabot, Appl. Catal. B Environ. 2018, 234, 10-18.

[51] Y. Liu, X. Liu, Q. Feng, D. He, L. Zhang, C. Lian, R. Shen, G. Zhao,
Y. Ji, D. Wang, et al., Adv. Mater. 2016, 28, 4747-4754.

[52] C. H. Cui, X. J. Liu, H. H. Li, M. R. Gao, H. W. Liang, H. Bin Yao, S. H. Yu, ChemCatChem 2012, 4, 1560-1563.

[53] N. Mahmood, C. Zhang, F. Liu, J. Zhu, Y. Hou, ACS Nano 2013, 7, 10307-10318.

[54] C. Lupu, J. G. Mao, J. W. Rabalais, A. M. Guloy, J. W. Richardson, Inorg. Chem. 2003, 42, 3765-3771.

[55] K. Kravchyk, L. Protesescu, M. I. Bodnarchuk, F. Krumeich, M. Yarema, M. Walter, C. Guntlin, M. V. Kovalenko, J. Am. Chem. Soc. 2013, 135, 4199-4202.

[56] R. Hu, Y. Ouyang, T. Liang, H. Wang, J. Liu, J. Chen, C. Yang, L. Yang, M. Zhu, Adv. Mater. 2017, 29, 1605006.

[57] Y. He, A. Li, C. Dong, C. Li, L. Xu, Chem. - A Eur. J. 2017, 23, 13724-13733.

[58] J. Qin, X. Zhang, N. Zhao, C. Shi, E. Liu, J. Li, C. He, RSC Adv. 2014, 4, 49247-49256.

[59] J. Qin, D. Liu, X. Zhang, N. Zhao, C. Shi, E. Z. Liu, F. He, L. Ma, Q. J. Qin, D. Liu, X. Zhang, N. Zhao, C. Shi, E. Z. Li
Li, J. Li, et al., Nanoscale 2017, 9, 15856-15864.

[60] Z. Wang, D. Wang, S. Luo, S. Bao, Y. Liu, X. Qi, C. He, C. Shi, N. Zhao, New J. Chem. 2016, 41, 393-402.

[61] C. Chen, Y. Wen, X. Hu, X. Ji, M. Yan, L. Mai, P. Hu, B. Shan, Y. Huang, Nat. Commun. 2015, 6, 6929.

[62] T.-C. Liu, J. Electrochem. Soc. 1998, 145, 1882.

[63] X. Xu, S. Ji, M. Gu, J. Liu, ACS Appl. Mater. Interfaces 2015, 7 , 20957-20964.

[64] X. Xu, J. Liu, J. Liu, L. Ouyang, R. Hu, H. Wang, L. Yang, M. Zhu, Adv. Funct. Mater. 2018, 28, 1707573.

\section{TOC graph}

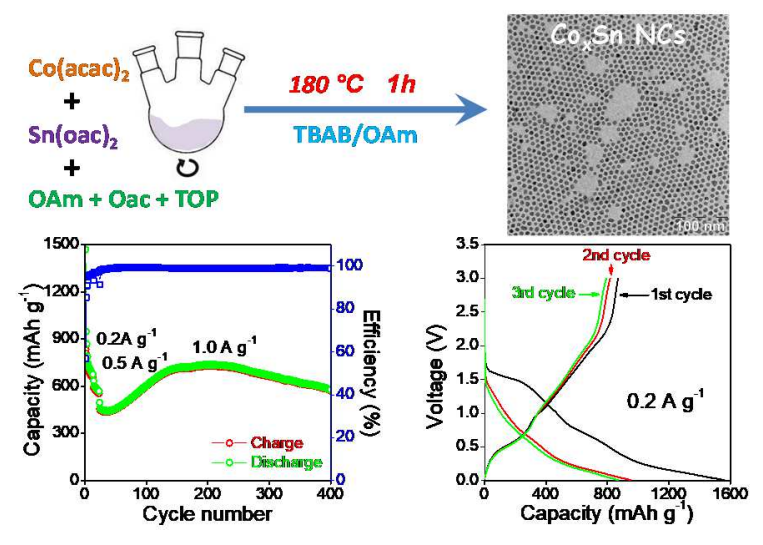

Colloidal Co-Sn nanoparticles as the application of lithium ion batteries. 